\title{
POTENTIAL THEORY IN THE GEOMETRY OF MATRICES
}

\author{
BY \\ JOSEPHINE MITCHELL( $(1)$
}

1. Introduction. Let $z=\left(z^{i k}\right)(1 \leqq j, k \leqq n)$ be a square matrix of complex numbers $z^{j k}, z^{*}$ the conjugate transpose of $z, 1$ the identity matrix, and 0 the zero matrix. We study the set $D$ defined by the relation $1-z z^{*}>0$, that is, the set of matrices $z$ such that the quadratic form $1-z z^{*}$ is positive definite $\left({ }^{2}\right)$. By defining a neighborhood of a point $z$, for example, as the set of points $y$ such that $\left|y^{j k}-z^{j k}\right|<\epsilon$ for arbitrary positive $\epsilon(1 \leqq j, k \leqq n)$, it can be shown that the set $D$ forms a convex bounded domain embedded in $2 n^{2}$ dimensional Euclidean space. If $z$ is a symmetric matrix (that is, $z=z^{\prime}$, where $z^{\prime}$ is the transpose of $z$ ), the corresponding domain $D$ lies in $n(n+1)$ dimensional Euclidean space and if $z$ is skew-symmetric $\left(z=-z^{*}\right)$ in $n(n-1)$ dimensional Euclidean space. We consider the first case although many of our results apply with only minor changes to the other cases as well. A proper part of the boundary of $D$ is the set of unitary matrices, that is, the set $B=\left[z z^{*}=1\right]$ $[9, \S 3]\left({ }^{3}\right)$. These three domains form part of a set of six irreducible domains possessing the property that all other bounded simple symmetric analytic spaces can be derived from them by analytic mappings and topological products $[7 ; 13]$. Of the other three irreducible domains two occur only for special values of $n$ and the sixth is the set of complex spheres.

The set of transformations which takes the domain $D$ into itself is

$$
w=(a z-a t)\left(d-d t^{*} z\right)^{-1},
$$

where $t$ is a fixed point of $D$ and $a^{*} a=\left(1-t t^{*}\right)^{-1}, d^{*} d=\left(1-t^{*} t\right)^{-1}$, and $a^{*} a t=t d^{*} d[13]$; the invariant metric with respect to this set of transformations is given by

$$
d s^{2}=\sigma\left(\left(1-z z^{*}\right)^{-1} d z\left(1-z^{*} z\right)^{-1} d z^{*}\right),
$$

where $\sigma$ is the trace of the matrix [7]. The domain $D$ with its metric is a Riemannian space.

In this paper we set up a potential theory for the domain $D$ with respect to the boundary $B$. In $\$ 2$ we derive the first and second (Laplacian) order differential parameters corresponding to the invariant metric (1.2). The Laplace equation is

Presented to the Society, May 1, 1954; received by the editors August 11, 1954.

(1) This research was done with the aid of a grant from the National Science Foundation.

(2) The symbol " $z$ " here stands for both the matrix $z$ and the point $z$, since it is always clear from the context which is meant.

(8) The notation $[\cdots]$ means the set of $z$ 's such that the property in the brackets holds. 


$$
\Delta v=4\left(\delta_{j}^{p}-z^{p l} \bar{z}^{j l}\right)\left(\delta_{q}^{k}-\bar{z}^{l k} z^{l q}\right) \partial^{2} v / \partial \bar{z}^{j k} \partial z^{p q}=0
$$

$(\bar{z}$ is the conjugate of $z$ ), where a repeated index means summation with respect to that index. Any real function with continuous second order partial derivatives (that is, any regular function) which satisfies this equation is said to be harmonic. Some of our results concern a proper subset (for $n>1$ ) of this class of functions, namely, those functions, called $B$-harmonic functions [2], which are the real or imaginary parts of complex analytic functions of several variables. A necessary and sufficient condition that a regular function $v$ be $B$-harmonic is that it satisfy the differential system

$$
\partial^{2} v / \partial \bar{z}^{j k} \partial z^{p q}=0
$$$$
(1 \leqq j, k, p, q \leqq n)
$$

[3]. The Laplacian (1.3) is invariant with respect to the set of transformations (1.1). In $\$ 3$ we derive Poisson's integral and function for $B$-harmonic functions from Cauchy's formula which has already been obtained by $\mathrm{S}$. Bochner [4]. The Poisson's function is harmonic but not $B$-harmonic. Using Poisson's integral, a Dirichlet problem is solved in $\$ 4$. In $\$ 5$ we obtain that solution of (1.3) and (1.4) which is a function of det $z z^{*}$ (the solution turns out to be the same for both differential systems). That the solution is a function of det $z z^{*}$ is in analogy with the classical Laplace equation and the fact that the Bergman and Szegö kernel functions in our case are known to be functions of $\operatorname{det}\left(1-z t^{*}\right)$, where $t$ is a fixed point of $D[9,4]$. Having obtained this function it is not difficult to define a unique Green's function of the differential system (1.4) with the required properties. In $\$ 6$ the normal plane to the boundary $B$ is set up in order that a Neumann's function of the differential system (1.4) can be defined for the domain $D$ and boundary $B$. These three domain functions are found to have many of the properties possessed by the same functions of the classical Laplace equation $\partial^{2} v / \partial z \partial \bar{z}=0$ for the unit circle $z \bar{z} \leqq 1$.

It seems to me that similar results could be obtained for more general Riemannian spaces if the space possessed a suitable boundary of dimension $n^{2}$. In this connection see [2]. We note that the set $B=\left[z z^{*}=1\right]$ is not a distinguished boundary surface of the kind usually considered Bergman [2]. The

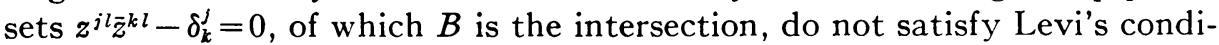
tion and hence are not analytic hypersurfaces [3]. A more general potential theory for Riemannian and Kähler manifolds with respect to the complete boundary is given in $[6 ; 16]$.

2. The first order differential parameter and the Laplacian of the invariant metric.

2.1. The Laplacian for a Hermitian metric. Let

$$
d s^{2}=\beta_{\mu \nu} d z^{\mu} d z^{\nu} \quad(1 \leqq \mu, \nu \leqq 2 n)
$$

be the square of a Hermitian metric, that is, (2.1) is a positive definite quadratic differential form with 


$$
\begin{aligned}
\beta_{j k} & =\beta_{j+n, k+n}=0, \\
\beta_{j, k+n} & =\beta_{k+n, j}=\bar{\beta}_{j+n, k}=\bar{\beta}_{k, j+n} \quad(1 \leqq j, k \leqq n)
\end{aligned}
$$

[15]. Let $\operatorname{det} \beta$ be the determinant of the matrix $\beta=\left(\beta_{\mu \nu}\right)$ and $\left(\beta^{\nu \mu}\right)$ the inverse matrix, so that

$$
\beta_{\rho \mu} \beta^{\rho \nu}=\delta_{\mu}^{\prime} \text {. }
$$

THEOREM 2.1. For the Hermitian metric (2.1) the Laplacian of a real function $V$ is

$$
\Delta V=2 \operatorname{det}^{-1 / 2} \beta \operatorname{Re}\left\{\frac{\partial}{\partial z^{j+n}}\left[\operatorname{det}^{1 / 2} \beta \beta^{j+n, k} \frac{\partial V}{\partial z^{k}}\right]\right\} \quad(1 \leqq j, k \leqq n) .
$$

"Re $f$ " is the real part of the function $f$ and "Im $f$ " the imaginary part.

Proof. Let the real form of the Hermitian metric (2.1) be given by

$$
d s^{2}=\alpha_{\mu \nu} d x^{\mu} d x^{\nu}, \quad \alpha_{\mu \nu}=\alpha_{\nu \mu},
$$

where

$$
\begin{aligned}
d z^{j} & =d x^{j}+i d x^{j+n}, & d z^{j+n} & =d x^{j}-i d x^{j+n}, \\
d x^{j} & =(1 / 2)\left(d z^{j}+d z^{j+n}\right), & d x^{j+n} & =(1 / 2 i)\left(d z^{j}-d z^{j+n}\right) .
\end{aligned}
$$

Corresponding to the square of the metric (2.5) the Laplacian of a real function $V$ is

$$
\Delta V=\operatorname{det}^{-1 / 2} \alpha \frac{\partial}{\partial x^{\mu}}\left(\operatorname{det}^{1 / 2} \alpha \alpha^{\mu \nu} \frac{\partial V}{\partial x^{\nu}}\right)
$$

[14], where $\left(\alpha^{\nu \mu}\right)=\alpha^{-1}$.

Introducing the operators

$$
\begin{aligned}
\frac{\partial}{\partial z^{j}} & =\frac{1}{2}\left(\frac{\partial}{\partial x^{j}}-\frac{i \partial}{\partial x^{j+n}}\right), & \frac{\partial}{\partial z^{j+n}} & =\frac{1}{2}\left(\frac{\partial}{\partial x^{j}}+\frac{i \partial}{\partial x^{j+n}}\right), \\
\frac{\partial}{\partial x^{j}} & =\frac{\partial}{\partial z^{j}}+\frac{\partial}{\partial z^{j+n}}, & \frac{\partial}{\partial x^{j+n}} & =i\left(\frac{\partial}{d z^{j}}-\frac{\partial}{d z^{j+n}}\right),
\end{aligned}
$$

we find that $\alpha_{\mu \nu}$ and $\alpha^{\mu \nu}$ transform according to the usual formulas

$$
\alpha_{\mu \nu}=\frac{\partial z^{\gamma}}{\partial x^{\mu}} \frac{\partial z^{\delta}}{\partial x^{\nu}} \beta_{\gamma \delta}, \quad \alpha^{\mu \nu}=\frac{\partial x^{\mu}}{\partial z^{\gamma}} \frac{\partial x^{\nu}}{\partial z^{\delta}} \beta^{\gamma \delta},
$$

Thus by formulas (2.2) and (2.9) we find that

$$
\alpha_{j k}=\alpha_{j+n, k+n}=2 \operatorname{Re} \beta_{j, k+n}, \quad \alpha_{j, k+n}=-\alpha_{j+n, k}=2 \operatorname{Im} \beta_{j, k+n} .
$$

Also

$$
\operatorname{det} \beta \beta^{j k}= \pm \operatorname{det}\left(\begin{array}{cc}
0 & A_{j} \\
B_{k} & 0
\end{array}\right)
$$


where $A_{j}$ equals $\left(\beta_{j, k+n}\right)$ with the $j$ th row omitted and $B_{k}$ equals $\left(\beta_{j+n, k}\right)$ with the $k$ th column omitted. By the Laplace expansion using the last $n$ columns we get zero since $A_{j}$ has only $n-1$ rows. Similarly for $\beta^{j+n, k+n}$. Thus

$$
\beta^{j k}=\beta^{i+n, k+n}=0 .
$$

Also, $\beta^{i, k+n}=\bar{\beta}^{i+n, k}$. Thus by formulas (2.9)

$$
\begin{aligned}
\alpha^{j k} & =\alpha^{j+n, k+n}=(1 / 2) \operatorname{Re} \beta^{j, k+n}, \\
\alpha^{j, k+n} & =-\alpha^{j+n, k}=-(1 / 2) \operatorname{Im} \beta^{j, k+n} .
\end{aligned}
$$

Also by $(2.10)$

$$
\operatorname{det} \alpha=\operatorname{det}\left(\begin{array}{cc}
\left(2 \operatorname{Re} \beta_{j, k+n}\right) & \left(2 \operatorname{Im} \beta_{j, k+n}\right) \\
\left(-2 \operatorname{Im} \beta_{j, k+n}\right) & \left(2 \operatorname{Re} \beta_{j, k+n}\right)
\end{array}\right) .
$$

To evaluate this determinant multiply the last $n$ columns by $i$ and add to the first $n$ columns; then multiply the first $n$ rows by $i$ and subtract from the last $n$ rows. This gives

$$
\operatorname{det} \alpha=\operatorname{det}\left(\begin{array}{cc}
\left(2 \beta_{j, k+n}\right) & \left(2 \operatorname{Im} \beta_{j, k+n}\right) \\
0 & \left(2 \bar{\beta}_{j, k+n}\right)
\end{array}\right) .
$$

Laplace's expansion used on the first $n$ columns gives

$$
\operatorname{det} \alpha=\operatorname{det} 2 \beta_{j, k+n} \operatorname{det} 2 \bar{\beta}_{j, k+n}=(-1)^{n} 2^{2 n} \operatorname{det} \beta,
$$

since by $(2.2)$

$$
\begin{aligned}
\operatorname{det} \beta & =\operatorname{det}\left(\begin{array}{cc}
0 & \left(\beta_{j, k+n}\right) \\
\left(\beta_{j+n, k}\right) & 0
\end{array}\right)=\operatorname{det}\left(\begin{array}{cc}
0 & \left(\beta_{j, k+n}\right) \\
\left(\bar{\beta}_{j, k+n}\right) & 0
\end{array}\right) \\
& =(-1)^{n} \operatorname{det} \beta_{j, k+n} \operatorname{det} \bar{\beta}_{j, k+n},
\end{aligned}
$$

which is real.

By means of (2.8) we transform (2.7) into complex form. There are four different types of terms to consider, viz.

$$
\begin{aligned}
\Delta V=\operatorname{det}^{-1 / 2} & \alpha X\left\{\left(\frac{\partial}{\partial z^{j}}+\frac{\partial}{\partial z^{j+n}}\right)\left[A\left(\frac{\partial}{\partial z^{k}}+\frac{\partial}{\partial z^{k+n}}\right) V\right]\right. \\
+ & \left(\frac{\partial}{\partial z^{j}}+\frac{\partial}{\partial z^{j+n}}\right)\left[-B i\left(\frac{\partial}{\partial z^{k}}-\frac{\partial}{\partial z^{k+n}}\right) V\right] \\
+ & i\left(\frac{\partial}{\partial z^{j}}-\frac{\partial}{\partial z^{j+n}}\right)\left[B\left(\frac{\partial}{\partial z^{k}}+\frac{\partial}{\partial z^{k+n}}\right) V\right] \\
& \left.-\left(\frac{\partial}{\partial z^{j}}-\frac{\partial}{\partial z^{j+n}}\right)\left[A\left(\frac{\partial}{\partial z^{k}}-\frac{\partial}{\partial z^{k+n}}\right) V\right]\right\}
\end{aligned}
$$

wherè by $(2.7),(2.12)$, and (2.14) 


$$
\begin{aligned}
& A=\operatorname{det}^{1 / 2} \alpha \alpha^{j k}=\operatorname{det}^{1 / 2} \alpha \alpha^{j+n, k+n}=(-1)^{n / 2} 2^{n-1} \operatorname{det}^{1 / 2} \beta \operatorname{Re} \beta^{j, k+n}, \\
& B=\operatorname{det}^{1 / 2} \alpha \alpha^{j+n, k}=-\operatorname{det}^{1 / 2} \alpha \alpha^{j, k+n}=(-1)^{n / 2} 2^{n-1} \operatorname{det}^{1 / 2} \beta \operatorname{Im} \beta^{j, k+n} .
\end{aligned}
$$

Thus

$$
\begin{gathered}
\Delta V=\operatorname{det}^{-1 / 2} \beta\left[\frac{\partial}{\partial z^{j}}\left(\operatorname{det}^{1 / 2} \beta \beta^{j, k+n} \frac{\partial V}{\partial z^{k+n}}\right)\right. \\
\left.+\frac{\partial}{\partial z^{j+n}}\left(\operatorname{det}^{1 / 2} \beta \bar{\beta}^{j, k+n} \frac{\partial V}{\partial z^{k}}\right)\right],
\end{gathered}
$$

which proves Theorem 2.1 since $\operatorname{det} \beta$ is real.

In order to simplify the notation we set

$$
2 \beta_{j, k+n}=g_{j k}, \quad 2 \beta_{j+n, k}=\bar{g}_{j k} .
$$

Then (2.1) becomes

$$
d s^{2}=g_{j k} d z^{i} d \bar{z}^{k}, \quad g_{j k}=\bar{g}_{k j} \quad(1 \leqq j, k \leqq n) .
$$

By (2.15),

$$
\operatorname{det} \beta=(-1)^{n} 2^{-2 n} \operatorname{det} g \operatorname{det} \bar{g},
$$

where $g=\left(g_{j k}\right)$. We also need $\beta^{i+n, k}$ in terms of $g$ 's. By (2.2) and (2.3)

$$
\delta_{j}^{k}=\beta_{\rho j} \beta^{\rho k}=\beta_{l+n, j} \beta^{l+n, k}=(1 / 2) \bar{g}_{l j} \beta^{l+n, k} \text {. }
$$

Thus

$$
\beta^{l+n, j}=2 \bar{g}^{l j},
$$

where $g^{-1}=\left(g^{k j}\right)$, and $\Delta V$ in terms of $g^{\prime}$ s becomes

$$
\Delta V=4 \operatorname{det}^{-1 / 2} g \bar{g} \operatorname{Re} \frac{\partial}{\partial \bar{z}^{j}}\left(\operatorname{det}^{1 / 2} g \bar{g} g^{k j} \frac{\partial V}{\partial z^{k}}\right) .
$$

Consequently

Corollary 2.1. The Laplacian of the Hermitian metric given by (2.20) is (2.22).

2.2. The Laplacian of the domain $D$.

Theorem 2.2. The Laplacian of the domain $D$ corresponding to the metric defined by (1.2) is

$$
\Delta=4\left(\delta_{j}^{p}-z^{p l} \bar{z}^{j l}\right)\left(\delta_{q}^{k}-\bar{z}^{l k} z^{l q}\right) \frac{\partial^{2}}{\partial \bar{z}^{j k} \partial z^{p q}} .
$$

The following result is needed in the proof of this theorem.

Lemma 2.1. The Jacobian of the square of the metric defined by (1.2) is $\operatorname{det}^{-2 n}\left(1-z z^{*}\right)$. 
Proof. If we set

$$
\begin{array}{ll}
R=\left(r_{j k}\right)=\left(1-z z^{*}\right)^{-1}, & S=\left(s_{j k}\right)=\left(1-z^{*} z\right)^{-1}, \\
r^{\prime}=\left(r^{k j}\right)=1-z z^{*}, & s^{\prime}=\left(s^{k j}\right)=1-z^{*} z^{(4)},
\end{array}
$$

then (1.2) becomes

$$
d s^{2}=r_{j k} d z^{k l} s_{l m} d \bar{z}^{j m}
$$

The matrix of this transformation is

$$
\left(\begin{array}{cccc}
r_{11} S & r_{21} S & \cdots & r_{n 1} S \\
\vdots & \vdots & \vdots \\
r_{1 n} S & r_{2 n} S & \cdots & r_{n n} S
\end{array}\right)
$$

which is the right direct product of the matrices $R^{\prime}$ and $S$ [8]. The value of the determinant of such a matrix is $\operatorname{det}^{n} R \operatorname{det}^{n} S$, which equals $\operatorname{det}^{-2 n}$ $\left(1-z z^{*}\right)$, since $\operatorname{det}\left(1-z^{*} z\right)=\operatorname{det}\left(1-z z^{*}\right)$ and $\operatorname{det}^{-1} a=\operatorname{det} a^{-1}$.

Proof of Theorem 2.2. In the notation of Corollary 2.1, $d s^{2}=g_{p q u v} d z^{p q} d \bar{z}^{u v}$ and comparison with (2.24) gives $g_{p q u v}=r_{u p} s_{q v}$. For the Laplacian $\Delta$ we need $g^{p q u v}$ where $g_{p q u v} g^{p q j k}=\delta_{u}^{j} \delta_{v}^{k}$. Now $r_{u p} r^{j p}=\delta_{u}^{j}$ and $s_{q v} s^{q k}=\delta_{v}^{k}$. Thus

$$
g^{p q j k}=r^{j p} s^{q k}=\left(\delta_{j}^{p}-z^{p l} \bar{z}^{j l}\right)\left(\delta_{q}^{k}-\bar{z}^{l k} z^{l q}\right) .
$$

Thus $\Delta V$ of (2.24) becomes

$$
\Delta V=4 \operatorname{det}^{2 n} r \operatorname{Re} \frac{\partial}{\partial \bar{z}^{j k}}\left(\operatorname{det}^{-2 n} r r^{i p^{q k}} \frac{\partial V}{\partial z^{p q}}\right)
$$

where $r^{j p_{S}{ }^{q k}}$ and $r$ are defined by (2.23). Using the differentiation formula

$$
d(\operatorname{det} x)=\operatorname{det} x \sigma\left(d x x^{-1}\right)(5),
$$

for nonsingular matrices $x$, we get that $\Delta V$ equals $4 \operatorname{det}^{2 n} r$ times the real part of

$$
\begin{aligned}
\operatorname{det}^{-2 n} r\left[r^{j p} s^{q k} \frac{\partial^{2} V}{\partial \bar{z}^{j k} \partial z^{p q}}-2 n \sigma(\right. & \left.-\frac{z \partial z^{*}}{\partial \bar{z}^{j k}} R\right) r^{j p S^{q k}} \frac{\partial V}{\partial z^{p q}} \\
& \left.-\left(n z^{p s^{q k}}+n r^{j p^{j q}}\right) \frac{\partial V}{\partial z^{p q}}\right] .
\end{aligned}
$$

The second last term equals

(4) $1-z z^{*}>0$ implies that det $\left(1-z z^{*}\right)>0$. Also, $1-z z^{*}>0$ and $1-z^{*} z>0$ define the same domain [4].

(5) Proof of (2.27). We have that $d(\operatorname{det} x)=\sum_{k=1}^{n}\left(d x^{1 k} X_{1 k}+\cdots+d x^{n k} X_{n k}\right)=\sigma\left(d x\left(X_{n k}\right)\right)$, where $X_{i k}$ is the cofactor of $x^{j k}$. For nonsingular $x,\left(X_{j k}\right)=x^{-1} \operatorname{det} x$, from which (2.27) follows. 


$$
\begin{aligned}
2 n z^{\alpha \beta} \frac{\partial \bar{z}^{\gamma \beta}}{\partial \bar{z}^{j k}} r_{\gamma \alpha} r^{j p^{q k}} & \frac{\partial V}{\partial z^{p q}} \\
& =2 n z^{\alpha k} r_{j \alpha} r^{j p} s^{q k} \frac{\partial V}{\partial z^{p q}} \\
& =2 n \sigma\left[z\left(1-z^{*} z\right)\left(\frac{\partial V}{\partial z^{p q}}\right)^{\prime}\left(1-z z^{*}\right)\left(1-z z^{*}\right)^{-1}\right] \\
& =2 n \sigma\left(\left(z-z z^{*} z\right)\left(\frac{\partial V}{\partial z^{p q}}\right)^{\prime}\right)
\end{aligned}
$$

while the last term equals

$$
\begin{aligned}
-n \sigma\left[z\left(1-z^{*} z\right)\left(\frac{d V}{\partial z^{p q}}\right)^{\prime}+\left(1-z z^{*}\right) z\right. & \left.\left(\frac{\partial V}{\partial z^{p q}}\right)^{\prime}\right] \\
& =-2 n \sigma\left[\left(z-z z^{*} z\right)\left(\frac{\partial V}{\partial z^{p q}}\right)^{\prime}\right],
\end{aligned}
$$

since $\sigma(a+b)=\sigma(a)+\sigma(b)$; the sum of the last two terms is zero, so that

$$
\Delta V=4 \operatorname{Re}\left[r^{i s^{q k}} \frac{\partial^{2} V}{\partial \bar{z}^{j k} \partial z^{p q}}\right] .
$$

The conjugate of this term is

$$
\left(\delta_{j}^{p}-\bar{z}^{p l} z^{j l}\right)\left(\delta_{q}^{k}-z^{l k} \bar{z}^{l q}\right) \frac{\partial^{2} V}{\partial z^{j k} \partial \bar{z}^{p q}},
$$

which upon interchanging $p$ and $j, q$ and $k$ gives the original expression. Thus $\Delta V$ is real for real $V$.

REMARKs. 1. For $n=1, \Delta V=4(1-z \bar{z})^{2} \partial^{2} V / \partial z \partial \bar{z}$ which corresponds to the metric $d s^{2}=(1-z \bar{z})^{-2} d z d \bar{z} / 4$.

2. The inner product form of $\Delta$ obtained by replacing $z^{*}$ by $t^{*}$ is

$$
\Delta\left(z, t^{*}\right)=4\left(\delta_{j}^{p}-z^{p l} \bar{t}^{j l}\right)\left(\delta_{q}^{k}-\bar{t}^{l k} z^{l q}\right) \frac{\partial^{2} V}{\partial \bar{t}^{j k} \partial z^{p q}} .
$$

3. Since for a complex analytic function $f, \partial f / \partial \bar{z}^{j k}=0(1 \leqq j, k \leqq n)$, we see that $\Delta f=0$. Also since the operator $\Delta$ is real, $\Delta \operatorname{Re} f=0$ and $\Delta \operatorname{Im} f=0$. Thus the class of $B$-harmonic functions is a subset of the set of harmonic functions.

2.3. Invariance of the Laplacian.

Theorem 2.3. The Laplacian (1.3) is invariant under the set of transformations (1.1). 
Proof. Under (1.1) or the equivalent transformation

$$
w=\left(a^{*}-z t^{*} a^{*}\right)^{-1}\left(z d^{*}-t d^{*}\right)
$$

[9], $\quad 1-w w^{*} \rightarrow\left(a^{*}-z t^{*} a^{*}\right)^{-1}\left(1-z z^{*}\right)\left(a-a t z^{*}\right)^{-1}=o^{-1} r^{\prime} o^{*-1}$, where $o=\left(o^{j k}\right)$ $=a^{*}-z t^{*} a^{*}$ and $r^{\prime}=1-z z^{*}$. Also, $1-w^{*} w \rightarrow\left(d^{*}-z^{*} t d^{*}\right)^{-1}\left(1-z^{*} z\right)\left(d-d t^{*} z\right)^{-1}$ $=p^{*-1} s^{\prime} p^{-1}$, where $p=d-d t^{*} z$ and $s^{\prime}=1-z^{*} z$.

Now

$$
\frac{\partial V}{\partial w^{p q}}=\frac{\partial V}{\partial z^{r s}} \frac{\partial z^{r s}}{\partial w^{p q}}
$$

But

$$
\frac{\partial z}{\partial w^{p q}}=\left(a^{*}-z t^{*} a^{*}\right) \frac{\partial w}{\partial w^{p q}}\left(d-d t^{*} z\right)=o\left(\frac{\partial w}{\partial w^{p q}}\right) p
$$

[9]. Thus

$$
\begin{aligned}
\frac{\partial^{2} V}{\partial \bar{w}^{j k} \partial w^{p q}} & =\frac{\partial^{2} V}{\partial \bar{z}^{u v} \partial z^{r s}} \frac{\partial z^{r s}}{\partial w^{p q}} \frac{\partial \bar{z}^{u v}}{\partial \bar{w}^{j k}} \\
& =\frac{\partial^{2} V}{\partial \bar{z}^{u v} \partial z^{r s}} o^{r a} \frac{\partial w^{a b}}{\partial w^{p q}} p^{b s} \bar{o}^{u c} \frac{\partial \bar{w}^{c d}}{\partial \bar{w}^{j k}} p^{d v} \\
& =\frac{\partial^{2} V}{\partial \bar{z}^{u v} \partial z^{r s}} o^{r p} p^{q s} \bar{o}^{u j} \bar{p}^{k v}
\end{aligned}
$$

Consequently

$$
\begin{aligned}
\Delta_{w} V & =4\left(\delta_{j}^{p}-w^{p l} \bar{w}^{j l}\right)\left(\delta_{q}^{k}-\bar{w}^{l k} w^{l q}\right) \frac{\partial^{2} V}{\partial \bar{w}^{j k} \partial w^{p q}} \\
& =4 o_{a p} r^{b a} \bar{o}_{b j} \bar{p}_{k c} c^{d c} p_{q d} o^{r p} p^{q s} \bar{o}^{u j} \bar{p}^{k v} \frac{\partial^{2} V}{\partial \bar{z}^{u v} \partial z^{r s}} .
\end{aligned}
$$

But $o_{a p} o^{r p}=\delta_{a}^{r}, \bar{o}_{b j} \bar{o}^{u j}=\delta_{b}^{u}, p_{k c} \bar{p}^{k v}=\delta_{c}^{v}, p_{q d} p^{q s}=\delta_{d}^{s}$, so that

$$
\Delta_{w} V=4\left(\delta_{u}^{r}-z^{r l} \bar{z}^{j l}\right)\left(\delta_{s}^{v}-\bar{z}^{l v} z^{l s}\right) \frac{\partial^{2} V}{\partial \bar{z}^{u v} \partial z^{r s}}=\Delta_{z} V .
$$

2.4. The first order differential parameter. For the metric defined by (2.5) the first order differential parameter is

$$
\Delta_{1} V=\alpha^{\mu \nu} \frac{\partial V}{\partial x^{\mu}} \frac{\partial V}{\partial x^{\nu}} .
$$

Transforming (2.38) into complex form by the methods of paragraph 2.1, we get 


$$
\Delta_{1} V=2 \operatorname{Re}\left(\beta^{j, k+n} \frac{\partial V}{\partial z^{j}} \frac{\partial V}{\partial z^{k+n}}\right)=4 \operatorname{Re}\left(g^{j k} \frac{\partial V}{\partial z^{j}} \frac{\partial V}{\partial \bar{z}^{k}}\right) .
$$

In particular for the domain $D$

$$
\Delta_{1} V=4\left(\delta_{j}^{p}-z^{p l} \bar{z}^{j l}\right)\left(\delta_{q}^{k}-\bar{z}^{l k} z^{l q}\right) \frac{\partial V}{\partial z^{p q}} \frac{\partial V}{\partial \bar{z}^{j k}} .
$$

2.5. The Euclidean volume element of the set $B$. The methods of paragraph 2.2 enable us to obtain the following results.

TheOREM 2.4. For the set $B=\left[z z^{*}=1\right]$ the Jacobian of the square of the Euclidean metric is $(-1)^{-n(n+1) / 2} \operatorname{det}^{-2 n} z$ and the Euclidean volume element is $(-1)^{-n(n+1) / 4} \operatorname{det}^{-n} z d z^{11} d z^{12} \cdots d z^{n n}$.

The second result of this theorem has already been obtained by Bochner by another method [4].

Proof. For matrices $z \in B, z^{*}=z^{-1}$. Thus $d z^{*}=-z^{-1} d z z^{-1}$, and the square of the Euclidean metric becomes

$$
\begin{aligned}
\dot{d s^{2}} & =\sigma\left(d z d z^{*}\right)=-\sigma\left(d z z^{-1} d z z^{-1}\right) \\
& =-d z^{k l} z_{j l} d z^{j m} z_{k m}=-z_{k m} z_{j l} d z^{k l} d z^{j m} .
\end{aligned}
$$

The matrix of this form is

$$
-\left(\begin{array}{cccc}
\left(z_{1 m} z_{1 l}\right) & \left(z_{1 m} z_{2 l}\right) & \cdots & \left(z_{1 m} z_{n l}\right) \\
\vdots & \vdots \\
\left(z_{n} z_{1 l}\right) & \left(z_{n m} z_{2 l}\right) & \cdots & \left(z_{n m} z_{n l}\right)
\end{array}\right)
$$

which can be reduced to the direct product of $-z^{-1}$ and $z^{-1}$ by means of elementary transformations. Interchange columns 2 and $n+1, \cdots, n$ and $n^{2}-n+1$. The first $n$ columns become $z_{11} z^{-1}, z_{21} z^{-1}, \cdots, z_{n 1} z^{-1}$. Then interchange colunns $n+3$ and $2 n+2, \cdots, 2 n$ and $n^{2}-n+2$. The second set of $n$ columns become $z_{12} z^{-1}, \cdots, z_{n 2} z^{-1}$. Repeating on each set of $n$ columns the final result is

$$
(-1)^{n-1+n-2+\cdots+1}\left(\begin{array}{l}
-z_{11} z^{-1} \cdots-z_{1 n} z^{-1} \\
-z_{n 1} z^{-1} \cdots-z_{n n} z^{-1}
\end{array}\right)
$$

which is $(-1)^{n(n-1) / 2}$ times the direct product of $-z^{-1}$ by $z^{-1}$, the value of whose determinant is $(-1)^{-n(n+1) / 2} \mathrm{det}^{-2 n} z$.

Now it is well known [14] that if the determinant of the square of the metric is $(-1)^{-n(n+1) / 2} \operatorname{det}^{-2 n} z$, then the determinant of the volume element is the square root of this expression, which completes the proof of Theorem 2.4. (According to Veblen this result holds for both real and imaginary coordinates.)

3. Poisson's integral and kernel $[17,18]$.

3.1. We need the following lemma for our proofs. 
Lemma 3.1. The matrix $1-z t^{*}$ is nonsingular for $t \in D$ and $z \in \bar{D}$ ( $\bar{D}$ is the closure of the domain $D$ ).

Proof. Suppose that $1-z t^{*}$ is singular, that is, det $\left(1-z t^{*}\right)=0$. By Cramer's rule there exists a nonzero vector $\lambda\left(\lambda^{1}, \cdots, \lambda^{n}\right)$ such that $\left(1-z t^{*}\right) \lambda=0$ or $1 \cdot \lambda=z t^{*} \lambda$. Thus $\lambda^{*} \lambda=\lambda^{*} t z^{*} z t^{*} \lambda$. Since $t \in D, \lambda^{*}\left(1-t t^{*}\right) \lambda>0$ or $\lambda^{*} \lambda$ $>\lambda^{*} t t^{*} \lambda$. Thus $0=\lambda^{*} \lambda-\lambda^{*} t z^{*} z t^{*} \lambda>\lambda^{*} t\left(1-z^{*} z\right) t^{*} \lambda$. Since $z \in \bar{D}\left({ }^{6}\right), 1-z^{*} z \geqq 0$, or $\lambda^{*} t\left(1-z^{*} z\right) t^{*} \lambda \geqq 0$, which gives a contradiction. Thus $\operatorname{det}\left(1-z t^{*}\right) \neq 0$ for $t \in D$ and $z \in \bar{D}$.

For $z \in B$ and $t \in D, z-t=\left(1-t z^{*}\right) z$ and $\operatorname{det}(z-t) \neq 0$ since $\operatorname{det} z \operatorname{det}$ $\left(1-t z^{*}\right) \neq 0$. The set of points $z$ for which det $(z-t)=0$ for fixed $t$ forms a $2 n^{2}-2$ dimensional manifold called the characteristic manifold of the function det $(z-t)=0$. (Since the function det $(z-t)$ is linear in each variable $z^{j k}$, its discriminant is 1 and the set defined by the equation has no singular points) [3].

3.2. For the unit circle $z \bar{z}=1$ the following relation exists between the Poisson's kernel $P(z, t)$ and the Szegö kernel function, $k(t, \bar{z})=[2 \pi(1-t \bar{z})]^{-1}$, [1], for all $t \bar{t}<1$. If $z=e^{i \phi}$, then

$$
P(z, t) d \phi=\frac{1}{2 \pi} \frac{1-t \bar{t}}{(z-t)(\bar{z}-\bar{t})} d \phi=\frac{1-t \bar{t}}{1-\overline{t z}} k(t, \bar{z}) \frac{d z}{i z} .
$$

By means of this relation Poisson's integral for a function harmonic in the unit circle (the real part of an analytic function for $n=1$ ) may be derived easily from Cauchy's formula. For if $f$ is analytic in $z \bar{z} \leqq 1$, the function $f^{x}(z)=f(z)(1-t \bar{t}) /(1-z \bar{t})$ is analytic in $z \bar{z} \leqq 1$; also, $f^{x}(t)=f(t)$. Thus by Cauchy's formula

$$
f(t)=f^{x}(t)=\frac{1}{2 \pi i} \int_{B} \frac{f(z)(1-t \bar{t})}{1-\bar{t} z} \frac{d z}{z-t}=\int_{0}^{2 \pi} f(z) P(z, t) d \phi .
$$

Since $P(z, t) d \phi$ is real, the real part of (3.2) gives Poisson's integral for the real part of the analytic function $f$.

This procedure may be generalized for the domain $D$ with $n>1$. Cauchy's formula exists for any function $f(z)$, analytic and bounded in $D$, and possessing boundary values in the ordinary sense at all points of $B[4]$ :

$$
f(t)=\frac{1}{V} \int_{B} \frac{f(z)}{\operatorname{det}^{n}\left(1-t z^{*}\right)} d V
$$

where

$$
d V=(-1)^{-n(n+1) / 4} \operatorname{det}^{-n} z d z^{11} d z^{12} \cdots d z^{n n},
$$

(6) $1-z z^{*}>0$ implies that $u\left(1-z z^{*}\right) u^{*}=u^{j}\left(1-z^{j l} \bar{z}^{k l}\right) \bar{u}^{k}$ is positive for any nonzero vector $u$. Now $z \in \bar{D}$ implies that $z=\lim z_{m}$ where $z_{m} \in D$. Thus $u\left(1-z_{m} z_{m}^{*}\right) u^{*}>0$ and since the form is continuous $u\left(1-z z^{*}\right) u^{*} \geqq 0$ for any vector $u$. 


$$
V^{-1}=\frac{1 ! 2 ! \cdots(n-1) !}{(2 \pi)^{n(n+1) / 2}}
$$

[11]. By Lemma 3.1 and the hypotheses on $f$ the function

$$
f^{x}(z)=\frac{\operatorname{det}^{n}\left(1-t t^{*}\right)}{\operatorname{det}^{n}\left(1-z t^{*}\right)} f(z)
$$

is analytic and bounded independently of $z$ in $D$ and has boundary values in the ordinary sense at all points of $B$. Also $f^{x}(t)=f(t)$. Thus by Cauchy's formula for the function $f^{x}$

$$
f(t)=f^{x}(t)=\frac{1}{V} \int_{B} \frac{f(z) \operatorname{det}^{n}\left(1-t t^{*}\right)}{\operatorname{det}^{n}\left(1-z t^{*}\right)\left(1-t z^{*}\right)} d V, \quad t \in D .
$$

The function

$$
P(z, t)=\frac{1}{V} \frac{\operatorname{det}^{n}\left(1-t t^{*}\right)}{\operatorname{det}^{n}\left(1-z t^{*}\right)\left(1-t z^{*}\right)}=\frac{1}{V} \frac{\operatorname{det}^{n}\left(1-t t^{*}\right)}{\operatorname{det}^{n}(z-t)\left(z^{*}-t^{*}\right)}
$$

is real. Hence taking the real part of (3.6) gives

$$
\operatorname{Re} f(t)=\int_{B} \operatorname{Re} f(z) P(z, t) d V, \quad t \in D .
$$

We call $P(z, t)$ the Poisson's function of the domain $D$. Thus

THEOREM 3.1. Let $f$ be an analytic bounded function in $D$ and defined on the boundary B. Poisson's integral for the real part of $f$ is given by (3.8) and Poisson's function of the domain $D$ by (3.7).

$P(z, t)$ has the following properties analogous to the case $n=1$.

Theorem 3.2. Poisson's function $P(z, t)$ is a harmonic function of $t$ for $t \in D$ and $z \in B$, that is,

$$
\Delta_{t} P(z, t)=0 \quad \text { for } t \in D \text { and } z \in B
$$

Also

$$
P(z, t)>0 \quad \text { for } t \in D \text { and } z \in \bar{D} \text {. }
$$

Proof. Since $t \in D, \operatorname{det}\left(1-t t^{*}\right)>0\left(^{4}\right)$. Also, by Lemma 3.1, $\operatorname{det}\left(1-z t^{*}\right) \neq 0$; consequently $\operatorname{det}\left(1-z t^{*}\right)\left(1-t z^{*}\right)=\operatorname{det}\left(1-z t^{*}\right) \operatorname{det}\left(1-t z^{*}\right)$ is positive. Thus $P(z, t)>0$ for $t \in D$ and $z \in \bar{D}$.

To simplify the notation in proving (3.9) set $r^{\prime}=1-t t^{*}, R=\left(1-t t^{*}\right)^{-1}$, $(z-t)^{-1}=W$ as in (2.23); thus

$$
A=(z-t)^{-1}\left(1-t t^{*}\right)\left(z^{*}-t^{*}\right)^{-1}=W r^{\prime} W^{*} .
$$

Then 


$$
\begin{aligned}
V \frac{\partial}{\partial t^{p q}} P(z, t) & =\frac{\partial}{\partial t^{p q}} \operatorname{det}^{n} A \\
& =n \operatorname{det}^{n} A \sigma\left[\frac{\partial}{\partial t^{p q}}\left(W r^{\prime} W^{*}\right)\left(W r^{\prime} W^{*}\right)^{-1}\right] \\
& =n \operatorname{det}^{n} A \sigma\left[\left(W \frac{\partial t}{\partial t^{p q}} W r^{\prime}-W \frac{\partial t}{\partial t^{p q}} t^{*}\right) R W^{-1}\right] \\
& =n \operatorname{det}^{n} A \sigma\left(\frac{\partial t}{\partial t^{p q}} W u^{\prime} R\right)
\end{aligned}
$$

since $\sigma(a b c)=\sigma(b c a)=\sigma(c a b)$ and $W r^{\prime}-t^{*}=(z-t)^{-1} \cdot\left(1-t t^{*}-z t^{*}+t t^{*}\right)$ $=(z-t)^{-1}\left(1-z t^{*}\right)=W u^{\prime}$, where $u^{\prime}=1-z t^{*}$. Similarly

$$
V \frac{\partial}{\partial \bar{t}^{j k}} \operatorname{det}^{n} A=n \operatorname{det}^{n} A \sigma\left(\bar{u} W^{*} \frac{\partial t^{*}}{\partial \bar{t}^{j k}} R\right) .
$$

Also

$$
\begin{aligned}
V \frac{\partial^{2} P(z, t)}{\partial \bar{t}^{j k} \partial t^{p q}}=n^{2} \operatorname{det}^{n} A \sigma\left(\frac{\partial t}{\partial t^{p q}} W u^{\prime} R\right) \sigma\left(\bar{u} W^{*} \frac{\partial t^{*}}{\partial \bar{t}^{j k}} R\right) \\
\quad+n \operatorname{det}^{n} A \sigma\left[\frac{\partial t}{\partial t^{p q}} W\left(-z \frac{\partial t^{*}}{\partial \bar{t}^{j k}} R+u^{\prime} R t \frac{\partial t^{*}}{\partial \bar{t}^{j k}} R\right)\right] .
\end{aligned}
$$

Forming $\Delta P(z, t)$ by means of (1.3) and (2.25) with $z$ replaced by $t$, the first term on the right of (3.14) gives

$$
\begin{aligned}
& 4 n^{2} \operatorname{det}^{n} A r^{i p_{S}}{ }^{q k} \frac{\partial t^{a b}}{\partial t^{p q}} w_{b c} u^{d c} \boldsymbol{r}_{d a} \bar{u}^{e f} \bar{w}_{g f} \frac{\partial \bar{t}^{h o}}{\partial \bar{t}^{j k}} r_{h e} \\
& =4 n^{2} \operatorname{det}^{n} A r^{j p} s^{q k} w_{q c} u^{d c} \boldsymbol{r}_{d p} \bar{u}^{e f} \bar{w}_{k j} \boldsymbol{r}_{j e} \\
& =4 n^{2} \operatorname{det}^{n} A \sigma\left(r^{\prime} r^{\prime-1} \bar{u} W^{*} s^{\prime} W u^{\prime} R\right) \\
& =4 n^{2} \operatorname{det}^{n} A \sigma\left(s^{\prime} W u^{\prime} R \bar{u} W^{*}\right) \\
& =4 n^{2} \operatorname{det}^{n} A \sigma\left[\left(1-t^{*} t\right)(z-t)^{-1}\left(1-z t^{*}\right)\left(1-t t^{*}\right)^{-1}\left(1-t z^{*}\right)\left(z^{*}-t^{*}\right)^{-1}\right]
\end{aligned}
$$

The second term on the right of (3.14) gives

$$
\begin{array}{rl}
4 n \operatorname{det}^{n} & A r^{j p_{S} q k} \frac{\partial t^{a b}}{\partial t^{p q}} w_{b c}\left(-z_{c d} \frac{\partial \bar{t}^{e d}}{\partial \bar{t}^{j k}} r_{e a}+u^{d c} \boldsymbol{r}_{d e} e^{e f} \frac{\partial \bar{t}^{g f}}{\partial \bar{t}^{j k}} \boldsymbol{r}_{g a}\right) \\
& =4 n \operatorname{det}^{n} A r^{j p^{q}{ }^{q k}} w_{q c}\left(-z_{c k} r_{j p}+u^{d c} \boldsymbol{r}_{d e} e^{e k} \boldsymbol{r}_{j p}\right) \\
& =4 n \operatorname{det}^{n} A \sigma\left(\boldsymbol{r}^{-1} \boldsymbol{r}\right) \sigma\left(-s^{\prime} W z+s^{\prime} W u^{\prime} R t\right) \\
& =4 n^{2} \operatorname{det}^{n} A \sigma\left[\left(1-t^{*} t\right)(z-t)^{-1}\left(-z+\left(1-z t^{*}\right)\left(1-t t^{*}\right)^{-1} t\right)\right] .
\end{array}
$$

Combining (3.15) and (3.16) we have 


$$
\begin{aligned}
& 4 n^{2} \operatorname{det}^{n} A \sigma\left[\left(1-t^{*} t\right)(z-t)^{-1}\left(1-z t^{*}\right)\left(1-t t^{*}\right)^{-1}\right. \\
& \left.\quad \cdot\left(1-t z^{*}+t z^{*}-t t^{*}\right)\left(z^{*}-t^{*}\right)^{-1}-\left(1-t^{*} t\right)(z-t)^{-1} z\right] \\
& =4 n^{2} \operatorname{det}^{n} A \sigma\left[\left(1-t^{*} t\right)(z-t)^{-1}\left(1-z t^{*}-z z^{*}+z t^{*}\right)\left(z^{*}-t^{*}\right)^{-1}\right]=0,
\end{aligned}
$$

since $z z^{*}=1$ and $\sigma(0)=0$.

This completes the proof of Theorem 3.2. It may be shown from (3.14) that $P(z, t)$ does not satisfy conditions (1.4) and hence is not a $B$-harmonic function.

\section{A solution of a Dirichlet problem.}

THEOREM 4.1. Let $u(z)$ be a continuous real function defined on the set $B=\left[z z^{*}=1\right]$ such that $u(z)=u(1)$ whenever $\operatorname{det}(1-z)=0$. Then there exists $a$ function $f$ harmonic in $D$ with the property $\lim f(t)=u(1)$ as $t \rightarrow 1, t \in D$. Such a function $f$ is given by

$$
f(t)=\frac{1}{V} \int_{B} \frac{u(z) \operatorname{det}^{n}\left(1-t t^{*}\right)}{\operatorname{det}^{n}\left(1-z t^{*}\right)\left(1-t z^{*}\right)} d V, \quad t \in D .
$$

There is al most one B-harmonic functionf satisfying the conditions of the theorem.

Proof. Since it is permissible to interchange the operators $\Delta$ and $\int$, by Theorem 3.2

$$
\Delta_{t} f(t)=\int_{B} \Delta_{t} P(z, t) u(z) d V_{z}=0
$$

for $z \in B$ and $t \in D$, where $P(z, t)$ is the kernel of (4.1). Thus $f$ is harmonic for $t \in D$. If $f$ is $B$-harmonic and $f_{1}$ were another $B$-harmonic function taking the value $u$ on $B$, then $f-f_{1}$ would be a $B$-harmonic function with the value zero on $B$, hence by Poisson's integral would be identically zero.

It remains to show that

$$
\lim _{t \rightarrow 1} f(t)=\lim _{t \rightarrow 1} \int_{B} P(z, t) u(z) d V_{z}=u(1),
$$

The set $B$ defined by $z z^{*}=1$ is the set product of $n^{2}$ closed sets. Thus $B$ is closed. Also $B$ is bounded so that it is compact. Consequently the continuous function $u(z)$ is uniformly continuous on $B$, so that given $\epsilon / 2>0$ there exists a $\delta>0$ and independent of $z$ such that $\sigma\left(\left(z-z_{1}\right)\left(z^{*}-z_{1}^{*}\right)\right)<\delta$ implies $\mid u(z)$ $-u\left(z_{1}\right) \mid<\epsilon / 2$ if $z, z_{1} \in B$. In particular for $z_{1}$ such that $\operatorname{det}\left(1-z_{1}\right)=0$,

$$
|u(z)-u(1)|<\epsilon / 2 \text { if } \sigma\left(\left(z-z_{1}\right)\left(z^{*}-z_{1}^{*}\right)\right)<\delta \quad \text { and } \quad z, z_{1} \in B .
$$

For fixed $z_{1}$ such that $\operatorname{det}\left(1-z_{1}\right)=0$ the neighborhood $N\left(\delta, z_{1}\right)=\left[\sigma\left(\left(z-z_{1}\right)\right.\right.$ $\left.\left.\cdot\left(z^{*}-z_{1}^{*}\right)\right)<\delta\right]$ is open. Thus the union $N(\delta)$ of the sets $N\left(\delta, z_{1}\right)$ taken over the set $\left[\operatorname{det}\left(1-z_{1}\right)=0\right]$ is an open set of $z$ 's. Consequently its complement $M(\delta)$ is closed; also $z \in M(\delta)$ implies that $\sigma\left(\left(z-z_{1}\right)\left(z^{*}-z_{1}^{*}\right)\right) \geqq \delta$ for all $z_{1}$ with 
$\operatorname{det}\left(1-z_{1}\right)=0$. The set $C=C(\delta)=M(\delta) \cap B$ is closed and compact in $B$. Therefore for $z \in C$ the continuous function $|\operatorname{det}(1-z)|$ attains its minimum, $m=m(\delta)$, at some point of the set $C$. Since $\operatorname{det}(1-z) \neq 0$ on $C, m \neq 0$.

Now det $\left(1-t z^{*}\right)$ is a continuous function of $z$ and $t$, defined in (4.1) on the topological product of the sets $\bar{D}$ and $B: \bar{D} \times B$. The sets $\bar{D}$ and $B$ are both closed; thus the set $\bar{D} \times B$ is closed in $E_{p} \times E_{p}\left(p=2 n^{2}\right)$, where $E_{p}$ is Euclidean space of dimension $p$ [12]. But the set $\bar{D} \times B$ is also bounded. Thus $\bar{D} \times B$ is compact in $E_{p} \times E_{p}$. Thus $\operatorname{det}\left(1-t z^{*}\right)$ is uniformly continuous on $\bar{D} \times B$. Therefore given $m / 2>0$ there exists an $\eta_{1}=\eta_{1}(m)>0$, independent of $t$ and $z$, such that $\sigma\left(\left(t-t_{1}\right)\left(t^{*}-t_{1}^{*}\right)\right)<\eta_{1}$ and $\sigma\left(\left(z-z_{1}\right)\left(z^{*}-z_{1}^{*}\right)\right)<\eta_{1}$ implies $\left|\operatorname{det}\left(1-t z^{*}\right)-\operatorname{det}\left(1-t_{1} z_{1}^{*}\right)\right|<m / 2$ if $t \in \bar{D}$ and $z \in B$. In particular for $t_{1}=1$ and $z_{1}=z$ we get

$$
\left|\operatorname{det}\left(1-t z^{*}\right)-\operatorname{det}\left(1-z^{*}\right)\right|<m / 2 \text { for } \sigma\left((t-1)\left(t^{*}-1\right)\right)<\eta_{1} .
$$

Thus

$$
\begin{aligned}
\left|\operatorname{det}\left(1-t z^{*}\right)\right| & \geqq\left|\operatorname{det}\left(1-z^{*}\right)\right|-\left|\operatorname{det}\left(1-t z^{*}\right)-\operatorname{det}\left(1-z^{*}\right)\right| \\
& >m-m / 2 \text { for } \sigma\left((t-1)\left(t^{*}-1\right)\right)<\eta_{1} \text { and } t \in \bar{D}, z \in C .
\end{aligned}
$$

By the continuity of $\operatorname{det}\left(1-t t^{*}\right)$ at $t=1$ given $\rho>0$ there exists an $\eta_{2}=\eta_{2}(\rho)>0$ such that

$$
\left|\operatorname{det}\left(1-t t^{*}\right)\right|<\rho \quad \text { if } \sigma\left((t-1)\left(t^{*}-1\right)\right)<\eta_{2} \text {. }
$$

Take $\eta=\min \left(\eta_{1}, \eta_{2}\right)=\eta(\rho, m)$. Then since $\int_{B} P(z, t) d V=1$ by Poisson's integral, and $P(z, t)>0$, we have

$$
\begin{aligned}
|f(t)-u(1)|= & \left|\int[u(z)-u(1)] P(z, t) d V\right| \\
\leqq & \int_{N(z) \cap B}|u(z)-u(1)| P(z, t) d V \\
& +\frac{1}{V} \int_{C} \frac{|u(z)-u(1)| \operatorname{det}^{n}\left(1-t t^{*}\right)}{\left|\operatorname{det}^{n}\left(1-t z^{*}\right)\right|} d V \\
< & \frac{\epsilon}{2} \int_{B} P(z, t) d V+\frac{2^{n+1} M \rho^{n}}{m^{n}} \frac{1}{V} \int_{B} d V<\epsilon
\end{aligned}
$$

for $\sigma\left((t-1)\left(t^{*}-1\right)\right)<\eta(t \in D)$, if $|u(z)| \leqq M$ on $B$ and $\rho$ is chosen as less than or equal to $\epsilon^{1 / n} m M^{-1 / n} 2^{-1-n / 2}$. Thus $f(t) \rightarrow u(1)$ as $t \in D \rightarrow 1$.

REMARKS. 1. Theorem 4.1 remains valid for $t \rightarrow z_{0} \neq 1, z_{0} \in B$, if we assume that $u(z)=u\left(z_{0}\right)$ whenever $\operatorname{det}\left(1-z_{0} z^{*}\right)=0(z \in B)$.

2. Theorems which are less restrictive with respect to the function $u$ have been obtained for domains which are $n$-cylinders, that is, domains such that $\left[z^{1} z^{1 *}<1, \cdots, z^{n} z^{n *}<1\right]$ because the Poisson kernel for such domains is obtained in factored form, namely 


$$
P(z, t)=\frac{1}{(2 \pi)^{n}} \prod_{j=1}^{n} \frac{\left(1-t^{i} t^{j *}\right)}{\left(z^{j}-t^{j}\right)\left(z^{j *}-t^{j *}\right)},
$$

and may be dealt with more easily. The same remark applies to the $n$-dimensional hypersphere $x x^{\prime}=x^{1} x^{1}+\cdots+x^{n} x^{n}<1$, since in this case the Poisson function

$$
P(z, t)=(1 / V)\left(1-t t^{\prime}\right) /|x-t|^{n}
$$

is singular only at $x=t[5]$.

5. Green's function of the differential system (1.4) for the domain $D$.

5.1. A solution of the differential system (1.4) and the Laplace equation. For the hypersphere $z^{1} \bar{z}^{1}+\cdots+z^{n} \bar{z}^{n} \leqq 1$ and the corresponding Laplace equation $\partial^{2} V / \partial z^{1} \partial \bar{z}^{1}+\cdots+\partial^{2} V / \partial z^{n} \partial \bar{z}^{n}=0$, the functions of potential theory, for example, the Green and Neumann's function, are functions of the distance $r=\left[\left(z^{j}-t^{j}\right)\left(\bar{z}^{j}-\bar{t}^{j}\right)\right]^{1 / 2}$, where $t$ is some fixed point and $z$ a variable point of the hypersphere. Likewise the Bergman and Szegö kernel functions are related to $r$. In analogy to this and particularly since the Bergman and Szegö kernel functions for the domain $D$ and boundary $B$ are functions of $\operatorname{det}\left(1-z t^{*}\right)[4 ; 9]$, we pose the following question: What scalar function of $\operatorname{det}(z-t)\left(z^{*}-t^{*}\right)$ is a solution of the differential system (1.4) for the domain $D$ ? The following result is obtained.

Theorem 5.1. The solution of the differential system (1.4) and of the Laplace differential equation (1.3) which is a function of $r=\operatorname{det}^{1 / 2}(z-t)\left(z^{*}-t^{*}\right)$ is $\log \operatorname{det}(z-t)\left(z^{*}-t^{*}\right) ; t$ is a fixed point and $z$ a variable point of $D$ such that $\operatorname{det}(z-t) \neq 0$.

Proof. Let $f(r)$ be a function of $r$. Then

$$
\begin{aligned}
\frac{\partial f}{\partial z^{p q}} & =\frac{f^{\prime}}{r} \frac{\partial}{\partial z^{p q}} \operatorname{det}^{1 / 2}(z-t)\left(z^{*}-t^{*}\right) \\
& =\frac{f^{\prime}}{2}\left\{\frac{\operatorname{det}\left(z^{*}-t^{*}\right)}{\operatorname{det}(z-t)}\right\}^{1 / 2} \frac{\partial}{\partial z^{p q}} \operatorname{det}(z-t),
\end{aligned}
$$

where $f^{\prime}=d f / d r$; also

$$
\begin{aligned}
\frac{\partial^{2} f}{\partial \bar{z}^{j k} \partial z^{p q}}= & \frac{f^{\prime \prime}}{4} \frac{\partial}{\partial \bar{z}^{i k}} \operatorname{det}\left(z^{*}-t^{*}\right) \frac{\partial}{\partial z^{p q}} \operatorname{det}(z-t) \\
& +\frac{1}{4} \frac{f^{\prime}}{r} \frac{\partial}{\partial \bar{z}^{j k}} \operatorname{det}\left(z^{*}-t^{*}\right) \frac{\partial}{\partial z^{p q}} \operatorname{det}(z-t) \\
= & \frac{1}{4}\left(f^{\prime \prime}+\frac{f^{\prime}}{r}\right) \frac{\partial}{\partial \bar{z}^{j k}} \operatorname{det}\left(z^{*}-t^{*}\right) \frac{\partial}{\partial z^{p q}} \operatorname{det}(z-t) \\
= & \frac{1}{4}\left(f^{\prime \prime}+\frac{f^{\prime}}{r}\right) \bar{W}_{j k} W_{p q},
\end{aligned}
$$


where $W_{p q}$ is the adjoint of the $p q$ term in $z-t$ and similarly for $\bar{W}_{j k}$. Also

$$
\begin{aligned}
\Delta f & =\left(f^{\prime \prime}+\frac{f^{\prime}}{r}\right) r^{j p S^{q k}} W_{p q} \bar{W}_{j k} \\
& =\left(f^{\prime \prime}+\frac{f^{\prime}}{r}\right) r^{2} \sigma\left(\left(1-z z^{*}\right)\left(z^{*}-t^{*}\right)^{-1}\left(1-z^{*} z\right)(z-t)^{-1}\right),
\end{aligned}
$$

since $W=\left(W_{q p}\right)=(z-t)^{-1} \operatorname{det}(z-t)$ and similarly for $W^{*}$, and $\sigma(k A)$ $=k \sigma(A)$, if $k$ is a scalar. If $\operatorname{det}(z-t) \neq 0$, then

$$
\sigma\left(\left(1-z z^{*}\right)\left(z^{*}-t^{*}\right)^{-1}\left(1-z^{*} z\right)(z-t)^{-1}\right) \neq 0
$$

and $W_{p q} \neq 0$ for some $p q$. Thus $f^{\prime \prime}+f^{\prime} / r=0$ in order that either (1.3) or (1.4) be satisfied, and the general solution of this equation is $c \log r+k(c, k$ constants).

5.2. In analogy to the case of the equation $\partial^{2} V / \partial z^{1} \partial \bar{z}^{1}+\cdots+\partial^{2} V / \partial z^{n} \partial \bar{z}^{n}$ $=0$ we make the following definition [5]:

Let $t$ be a fixed point of the domain $D, z$ a variable point of $\bar{D}$, and $r=\operatorname{det}^{1 / 2}$ $(z-t)\left(z^{*}-t^{*}\right)$. A Green's function $G(z, t)$ of the differential system (1.4) for the domain $D$ is defined to be a real solution of (1.4) depending on $t$, which is zero on the boundary $B$ and which is such that $G(z, t)-f(r)$ is regular in $D$ and continuous in $\bar{D}$, where $f(r)$ is the function of Theorem 5.1 .

Then

Theовем 5.2. The Green's function of the differential system (1.4) for the domain $D$ is

$$
G(z, t)=2^{-1} \log \operatorname{det}\left(1-z t^{*}\right)\left(z^{*}-t^{*}\right)^{-1}(z-t)^{-1}\left(1-t z^{*}\right),
$$

where $t \in D$ and $z \in \bar{D}$. Under the conditions of the definition Green's function is unique.

Proof. By Lemma 3.1 the function $G(z, t)$ is regular for $z \in \bar{D}$ except where $\operatorname{det}(z-t)=0$. For $z \in B, 1-z t^{*}=z\left(z^{*}-t^{*}\right)$, so that $G(z, t)=2^{-1} \log \operatorname{det} z z^{*}$ $=0$. Writing

$$
G(z, t)=-2^{-1} \log \operatorname{det}(z-t)\left(z^{*}-t^{*}\right)+2^{-1} \log \operatorname{det}\left(1-z t^{*}\right)\left(1-t z^{*}\right),
$$

we see by (2.27) that

$$
2 \frac{\partial G}{\partial z^{p q}}=-\sigma\left(\frac{\partial z}{\partial z^{p q}}(z-t)^{-1}\right)-\sigma\left(\frac{\partial z}{\partial z^{p q}} t^{*}\left(1-z t^{*}\right)^{-1}\right)
$$

which is independent of $z^{*}$. Thus $G(z, t)$ satisfies the differential system (1.4). The Green's function is unique. Suppose that $G_{1}(z, t)$ is another Green's function. Then, $G(z, t)-G_{1}(z, t)$ is $B$-harmonic in $D$ and vanishes on $B$. Hence by Poisson's integral $G(z, t)-G_{1}(z, t)$ is identically zero in $D$.

Green's function is related to the Bergman kernel function 


$$
K\left(z, t^{*}\right)=\frac{1}{V_{D} \operatorname{det}^{2 n}\left(1-z t^{*}\right)},
$$

where $V_{D}$ is the volume of the domain $D$ [9], in the following way. Set

$$
\Delta_{0}\left(z, t^{*}\right)=\operatorname{det}^{-2 n}\left(1-z t^{*}\right) \Delta\left(z, t^{*}\right),
$$

where $\Delta\left(z, t^{*}\right)$ is defined by (2.32). Then

TheOREM 5.3. Green's function of the domain $D$ satisfies the following relations:

$$
\Delta\left(z, t^{*}\right) G(z, t)=-2 n^{2}
$$

and

$$
\Delta_{0}\left(z, t^{*}\right) G(z, t)=-2 n^{2} V_{D} K\left(z, t^{*}\right) .
$$

Proof. From (5.5) we have

$$
\begin{aligned}
2 \frac{\partial^{2} G}{\partial \bar{t}^{j k} \partial z^{p q}}=- & \sigma\left(\frac{\partial z}{\partial z^{p q}} \frac{\partial t^{*}}{\partial \bar{t}^{j k}}\left(1-z t^{*}\right)^{-1}+\frac{\partial z}{\partial z^{p q}} t^{*}\left(1-z t^{*}\right)^{-1}\right. \\
& \left.\cdot \frac{z \partial t^{*}}{\partial \bar{t}^{j k}}\left(1-z t^{*}\right)^{-1}\right) .
\end{aligned}
$$

Set $u^{\prime}=1-z t^{*}, v^{\prime}=1-t^{*} z, U=\left(1-z t^{*}\right)^{-1}$. Then by $(2.32)$

$$
\begin{aligned}
& 2 \Delta\left(z, t^{*}\right) G(z, t) \\
& =-4 u^{j p_{v} q k}\left(\frac{\partial z^{a b}}{\partial z^{p q}} \frac{\partial \bar{t}^{c b}}{\partial \bar{t}^{j k}} u_{c a}+\frac{\partial z^{a b}}{\partial z^{p q}} \bar{t}^{c b} u_{c d} z^{d e} \frac{\partial \bar{t}^{j e}}{\partial \bar{t}^{j k}} u_{f a}\right) \\
& =-4 u^{j p} v^{q k}\left(\delta_{q}^{k} u_{j p}+\pi^{c q} u_{c d} z^{d k} u_{j p}\right) \\
& =-4 \sigma\left\{\left(1-z t^{*}\right)\left(1-z t^{*}\right)^{-1}\left[\sigma\left(1-t^{*} z\right)+\sigma\left(\left(1-t^{*} z\right) t^{*}\left(1-z t^{*}\right)^{-1} z\right)\right]\right\} \\
& =-4 n \sigma\left[\left(1-t^{*} z\right)\left(1+t^{*}\left(1-z t^{*}\right)^{-1} z\right)\right] \text {, }
\end{aligned}
$$

since $\sigma(1)=n$. Now

$$
1+t^{*}\left(1-z t^{*}\right)^{-1} z=\left(1-t^{*} z\right)^{-1}
$$

so that $\Delta\left(z, t^{*}\right) G(z, t)=-2 n^{2}$ and (5.8) is proved. Relation (5.9) follows from (5.7).

6. Neumann's function of the differential system (1.4) for the domain $D$.

6.1 . For the case $n=1$ at each point of the boundary of the unit circle $z z^{*}<1$ there is a unique outer normal $n$ directed along the radius vector so that the differential operator $\partial / \partial n$ has meaning. The Neumann function $N(z, t)$ of a smoothly bounded domain $D$ with respect to a point $t$ of $D$ is defined to be a function of the form $N(z, t)=-\log |z-t|+N_{1}(z, t)$, where $N_{1}(z, t)$ is $B$-harmonic in $D$; on the boundary $B$ of $D, N(z, t)$ has a constant normal derivative, that is, 


$$
\frac{\partial N(z, t)}{\partial n}=-\frac{2 \pi}{L}
$$

for $z \in B$,

where $L$ is the length of $B . N(z, t)$ is further normalized by the condition $\int_{B} N(z, t)$ $d s=0[10]$.

By means of this definition it may be shown that the Neumann's function for $z \bar{z}<1$ is

$$
\begin{aligned}
N(z, t) & =-2^{-1} \log (z-t)(\bar{z}-\bar{t})-2^{-1} \log \left(z-\frac{1}{\bar{t}}\right)\left(\bar{z}-\frac{1}{t}\right)-2^{-1} \log t \bar{t} \\
& =-2^{-1} \log (z-t)(\bar{z}-\bar{t})(1-z \bar{t})(1-\bar{z} t) .
\end{aligned}
$$

We check that (6.1) holds for the function (6.2) where $L=2 \pi$. Using the operators (2.6) and (2.8), we have

$$
\begin{aligned}
\frac{\partial N(z, t)}{\partial n} & =\frac{\partial N}{\partial z} \frac{\partial z}{\partial n}+\frac{\partial N}{\partial \bar{z}} \frac{\partial \bar{z}}{\partial n} \\
& =\frac{-1}{2(z-t)(\bar{z}-\bar{t})}\left[(1-2 z \bar{t}+t \bar{t}) \bar{z} \frac{\partial z}{\partial n}+(1-2 t \bar{z}+t \bar{t}) z \frac{\partial \bar{z}}{\partial n}\right] .
\end{aligned}
$$

On $\left.z \bar{z}=1, \partial z / \partial n=\partial r e^{i \theta} / \partial r\right)_{r=1}=e^{i \theta}=z$ and $\partial \bar{z} / \partial n=\bar{z}$. Thus $\partial N / \partial n=-1$ on $z \bar{z}=1$ as was to be proved.

6.2. In order to define the Neumann function for $n>1$ we need the normal plane to the boundary $B$, defined by the condition $z z^{*}=1$ or by the set of $n^{2}$ real equations

$$
\begin{aligned}
& F^{j k}=\operatorname{Re} z^{j l} \bar{z}^{k l}-\delta_{k}^{j}=0 \\
& (j \leqq k), \\
& F^{k j}=\operatorname{Im} z^{j l \bar{z}^{k l}}=0 \\
& (j<k) \text {. }
\end{aligned}
$$

Each equation of (6.4) defines a real $2 n^{2}-1$ dimensional hypersurface. At a point $P$ on $B$ each hypersurface $F^{j k}=0(1 \leqq j, k \leqq n)$ has a unique normal $n^{j k}$, whose components are $\left[\partial F^{j k} / \partial x^{\mu q}\right](1 \leqq \mu \leqq 2 n, 1 \leqq q \leqq n)$, where

$$
x^{p q}=\left(z^{p q}+\bar{z}^{p q}\right) / 2, \quad y^{p q}=x^{p+n, q}=(1 / 2 i)\left(z^{p q}-\bar{z}^{p q}\right) \quad(1 \leqq p \leqq n)
$$

and whose direction cosines are

$$
\left[\frac{1}{N} \frac{\partial F^{j k}}{\partial x^{\mu q}}\right] \text { where } \quad N^{2}=\frac{\partial F^{j k}}{\partial x^{\mu q}} \frac{\partial F^{j k}}{\partial x^{\mu q}} \quad(j, k \text { fixed }) .
$$

It is easy to show that the normals $n^{j k}$ at $P$ are mutually perpendicular; also, $N=2$ if $j=k$, and $=2^{1 / 2}$ if $j \neq k$. Thus the set of normals determine a hyperplane of dimension $n^{2}$ which is normal to the surface $z z^{*}=1$.

The derivatives $\partial F^{j k} / \partial x^{\mu q}$ may be expressed in terms of $\partial x^{\mu q} / \partial n^{j k}$ as follows. Let $P\left(x^{\mu q}\right)$ be the point at which the unit normal $n^{j k}$ meets the hypersurface $F^{j k}=0$ and $Q\left(x^{\mu q}+\Delta x^{\mu q}\right)$ a neighboring point on $n^{j k}$. The direction numbers of $n^{j k}$ are $\left(\Delta x^{11}, \cdots, \Delta x^{2 n, n}\right)$ and the direction cosines $\left(\Delta x^{11} / \Delta n^{j k}\right.$ 
$\left.\cdots, \Delta x^{2 n, n} / \Delta n^{j k}\right)$ where $\Delta n^{j k}$ is an increment of length on $n^{j k}$. In the limit as $\Delta n^{j k} \rightarrow 0$ this gives the direction cosines of $n^{j k}$ as

$$
\left(\partial x^{11} / \partial n^{j k}, \cdots, \partial x^{2 n, n} / \partial n^{j k}\right) .
$$

Thus by the results of the last paragraph

$$
\frac{\partial x^{\mu q}}{\partial n^{j k}}=\frac{1}{N} \frac{\partial F^{j k}}{\partial x^{\mu q}} \quad(1 \leqq \mu \leqq 2 n, 1 \leqq j, k, q \leqq n),
$$

where $N=2$ if $j=k$ and $2^{1 / 2}$ if $j \neq k$.

We also need the derivatives $\partial z / \partial n^{j k}$ and $\partial z^{*} / \partial n^{j k}=\left(\partial z / \partial n^{j k}\right)^{*}$, which equal by (2.6), (2.8), and (6.5)

$$
\frac{\partial z^{p q}}{\partial n^{j k}}=\frac{\partial x^{p q}}{\partial n^{j k}}+i \frac{\partial x^{p+n, q}}{\partial n^{j k}}=\frac{1}{N}\left(\frac{\partial F^{j k}}{\partial x^{p q}}+i \frac{\partial F^{j k}}{\partial x^{p+n, q}}\right)=\frac{2}{N} \frac{\partial F^{j k}}{\partial \bar{z}^{p q}} .
$$

Instead of using the hypersurfaces defined by $F^{j k}=0$ and their normals $n^{i k}$ it is more convenient for us to use the forms

$$
\begin{aligned}
& f^{j k}=z^{j l} \bar{z}^{k l}-\delta_{k}^{j}=F^{j k}+i F^{k j}=0 \\
& f^{k j}=\bar{f}^{j k},
\end{aligned}
$$

and the unit "complex normals" $\eta^{j k}$ associated with them as follows:

$$
\eta^{i j}=n^{i j},
$$

$$
\eta^{j k}=\frac{1}{2^{1 / 2}}\left(n^{j k}-i n^{k j}\right), \quad \eta^{k j}=\bar{\eta}^{j k}=\frac{1}{2^{1 / 2}}\left(n^{j k}+i n^{k j}\right) \quad(j<k),
$$

plus the operators

(6.9) $\frac{\partial}{\partial \eta^{j k}}=\frac{1}{2} 2^{1 / 2}\left(\frac{\partial}{\partial n^{j k}}+\frac{i \partial}{\partial n^{k j}}\right), \frac{\partial}{\partial \eta^{k j}}=\frac{1}{2} 2^{1 / 2}\left(\frac{\partial}{\partial n^{j k}}-\frac{i \partial}{\partial n^{k j}}\right)(j<k)$.

Then by (6.9), (6.6), and (6.7),

$$
\frac{\partial z^{p q}}{\partial \eta^{j k}}=\frac{1}{2} 2^{1 / 2}\left(\frac{\partial z^{p q}}{\partial n^{j k}}+i \frac{\partial z^{p q}}{\partial n^{k i}}\right)=\frac{2 \cdot 2^{1 / 2}}{2 \cdot 2^{1 / 2}}\left(\frac{\partial F^{j k}}{\partial \bar{z}^{p q}}+i \frac{\partial F^{k j}}{\partial \bar{z}^{p q}}\right)=\frac{\partial f^{j k}}{\partial \bar{z}^{p q}}
$$

$$
\frac{\partial z^{p q}}{\partial \eta^{j j}}=\frac{\partial f^{j i}}{\partial \bar{z}^{p q}}, \quad \frac{\partial z^{p q}}{\partial \eta^{k j}}=\frac{\partial f^{k j}}{\partial \bar{z}^{p q}} .
$$

Also,

$$
\frac{\partial z^{*}}{\partial \eta^{j k}}=\left(\frac{\partial z}{\partial \eta^{k i}}\right)^{*}
$$

since 


$$
\frac{\partial \bar{z}^{q p}}{\partial \eta^{j k}}=\frac{1}{2} 2^{1 / 2}\left(\frac{\partial \bar{z}^{q p}}{\partial n^{j k}}+i \frac{\partial \bar{z}^{q p}}{\partial n^{k j}}\right)=\frac{1}{2} 2^{1 / 2}\left(\overline{\frac{\partial z^{q p}}{\partial n^{j k}}+i \frac{\partial z^{q p}}{\partial n^{k j}}}\right)=\frac{\overline{\partial z^{q p}}}{\partial \eta^{k j}} .
$$

The derivatives of $f^{j k}=\boldsymbol{z}^{j l} \overline{\mathbf{z}}^{k l}-\delta_{\boldsymbol{k}}^{j}=0(1 \leqq j, k \leqq n)$ are

$$
\frac{\partial f^{j k}}{\partial z^{p q}}=\delta_{p}^{j} \bar{z}^{k q}, \quad \frac{\partial f^{j k}}{\partial \bar{z}^{p q}}=\delta_{p}^{k} z^{j q} .
$$

Thus by (6.10) and (6.11)

$$
\frac{\partial z^{p q}}{\partial \eta^{j k}}=\delta \delta^{k} z^{j q}, \quad \frac{\partial \bar{z}^{p q}}{\partial \eta^{j k}}=\delta_{p}^{j} \bar{z}^{k q} .
$$

6.3. We now are able to prove the following theorem:

THEOREM 6.1. The function

$$
N(z, t)=-2^{-1} \log \operatorname{det}(z-t)\left(z^{*}-t^{*}\right)\left(1-z t^{*}\right)\left(1-t z^{*}\right)
$$

satisfies the differential system (1.4) for $z \in D, t \in D$ except when $\operatorname{det}(z-t)=0$. Alsú

$$
\left(\frac{\partial N(z, t)}{\partial \eta^{j k}}\right)=-1 \quad \text { for } z \in B, t \in D
$$

and

$$
\int_{B} N(z, t) d V_{z}=0
$$

Also log $\operatorname{det}\left(1-z t^{*}\right)\left(1-t z^{*}\right)$ is B-harmonic in $D$.

In analogy to the case $n=1$ and the definition of the Green's function we define $N(z, t)$ to be the Neumann's function of the domain $D$.

Proof. A calculation similar to (5.5) shows that $N(z, t)$ is $B$-harmonic for $z$ and $t \in D$ if $\operatorname{det}(z-t) \neq 0$. Now consider (6.15). Setting $u^{\prime}=\left(u_{k j}\right)=1-z t^{*}$, $w^{\prime}=z-t$, we have

$$
\begin{aligned}
2 \frac{\partial N}{\partial \eta^{j k}} & =-\frac{\partial}{\partial \eta^{j k}}\left(\log \operatorname{det} w^{\prime} \bar{w}+\log \operatorname{det} u^{\prime} \bar{u}\right) \\
& =-\sigma\left(\frac{\partial w^{\prime}}{\partial \eta^{j k}} w^{\prime-1}+\frac{\partial \bar{w}}{\partial \eta^{j k}} \bar{w}^{-1}\right)-\sigma\left(\frac{\partial u^{\prime}}{\partial \eta^{j k}} u^{\prime-1}+\frac{\partial \bar{u}}{\partial \eta^{j k}} \bar{u}^{-1}\right) \\
& =-\left(\frac{\partial z^{p q}}{\partial \eta^{j k}} w_{q p}+\frac{\partial \bar{z}^{q p}}{\partial \eta^{j k}} \bar{w}_{p q}-\frac{\partial z^{p q}}{\partial \eta^{j k}} \bar{t}^{r q} u_{r p}-t^{p q} \frac{\partial \bar{z}^{r q}}{\partial \eta^{j k}} \bar{u}_{p r}\right) .
\end{aligned}
$$

By (6.13) we have $p=k$ in the first and third terms, $q=j$ in the second term, and $r=j$ in the last term. In particular for $j=k$ 


$$
\begin{aligned}
2 \frac{\partial N}{\partial \eta^{j j}}= & -\left(z^{j q} w_{q j}+\bar{z}^{j p} \bar{w}_{p j}-z^{j q} \bar{t}^{r q} u_{r j}-t^{p q} \bar{z}^{j q} \bar{u}_{p j}\right) \\
= & - \text { the } j j \text { term of }\left(z w^{\prime-1}+\bar{w}^{-1} z^{*}-z t^{*} u^{\prime-1}-\bar{u}^{-1} t z^{*}\right) \\
= & - \text { the } j j \text { term of }\left[z z^{*}\left(1-t z^{*}\right)^{-1}+\left(1-z t^{*}\right)^{-1} z z^{*}-z t^{*}\left(1-z t^{*}\right)^{-1}\right. \\
& \left.-\left(1-t z^{*}\right)^{-1} t z^{*}\right] \\
= & - \text { the } j j \text { term of }\left[\left(1-t z^{*}\right)^{-1}\left(1-t z^{*}\right)+\left(1-z t^{*}\right)\left(1-z t^{*}\right)^{-1}\right] \\
= & -2
\end{aligned}
$$

For $j<k$ we have

$$
\begin{aligned}
2 \frac{\partial N}{\partial \eta^{j k}} & =-\left(z^{j q} w_{q k}+\bar{z}^{k p} \bar{w}_{p j}-z^{j q} \bar{t}^{r q} u_{r k}-t^{p q} \bar{z}^{k q} \bar{u}_{p j}\right) \\
& =- \text { the } j k \text { term of }\left(z w^{\prime-1}+\bar{w}^{-1} z^{*}-z t^{*} u^{\prime-1}-\bar{u}^{-1} t z^{*}\right) \\
& =- \text { the } j k \text { term of } 1=0 .
\end{aligned}
$$

Similarly $\partial N / \partial \eta^{k i}=0$ and (6.15) is proved.

Now consider $\int_{B} N(z, t) d V_{z}$. For $z \in B, z-t=\left(1-t z^{*}\right) z$. Thus $N(z, t)$ $=-\log \operatorname{det}\left(1-z t^{*}\right)\left(1-t z^{*}\right)$ on $B$. By Lemma 3.1, det $\left(1-z t^{*}\right) \neq 0$ for $t \in D$ and $z \in \bar{D}$. Therefore log det $\left(1-z t^{*}\right)\left(1-t z^{*}\right)$ is $B$-harmonic for $z \in \bar{D}$. Thus by Poisson's formula at $z=z_{0}$

$$
\begin{aligned}
\log \operatorname{det}\left(1-z_{0} t^{*}\right) & \left(1-t z_{0}^{*}\right) \\
= & \frac{1}{V} \int_{B} \frac{\operatorname{det}^{n}\left(1-z_{0} z_{0}^{*}\right) \log \operatorname{det}\left(1-z t^{*}\right)\left(1-t z^{*}\right)}{\operatorname{det}^{n}\left(z-z_{0}\right)\left(z^{*}-z_{0}^{*}\right)} d V,
\end{aligned}
$$

and in particular at $z_{0}=0$

$$
0=\frac{1}{V} \int_{B} \log \operatorname{det}\left(1-z t^{*}\right)\left(1-t z^{*}\right) d V=-\frac{1}{V} \int_{B} N(z, t) d V .
$$

6.4. Calculation of $\left(\partial G / \partial \eta^{j k}\right)$ on $B$. For $n=1$ there is the following connection between the normal derivative of the Green's function and Poisson's function on $z \bar{z}=1$.

$$
\frac{\partial G}{\partial n}=\frac{-(1-t \bar{t})}{(z-t)(\bar{z}-\bar{t})}=-2 \pi P(z, t) .
$$

For $n>1$ the following result can be proved:

Theorem 6.2. For Green's function $G(z, t)$ of the domain $D$ we have

$$
\left(\frac{\partial G}{\partial \eta^{j k}}\right)=-\left(1-t z^{*}\right)^{-1}\left(1-t t^{*}\right)\left(1-z t^{*}\right)^{-1} \quad \text { for } t \in D, z \in B,
$$

where the differential operators $\partial / \partial \eta^{j k}$ are defined by (6.9). 
Proof. Since $G(z, t)=2^{-1} \log \operatorname{det}\left(1-z t^{*}\right)\left(1-t z^{*}\right)-2^{-1} \log \operatorname{det}(z-t)$ $\cdot\left(z^{*}-t^{*}\right)$ and $N(z, t)=-2^{-1} \log \operatorname{det}(z-t)\left(z^{*}-t^{*}\right)-2^{-1} \log \operatorname{det}\left(1-z t^{*}\right)$ $\cdot\left(1-t z^{*}\right)$, we see that analogously to $(6.18)$ on $B$

$$
\begin{aligned}
2 \frac{\partial G}{\partial \eta^{j j}}= & - \text { the } j j \text { term of }\left[z(z-t)^{-1}+\left(z^{*}-t^{*}\right)^{-1} z^{*}\right. \\
& \left.+z t^{*}\left(1-z t^{*}\right)^{-1}+\left(1-t z^{*}\right)^{-1} t z^{*}\right] \\
= & - \text { the } j j \text { term of }\left[\left(1+z t^{*}\right)\left(1-z t^{*}\right)^{-1}+\left(1-t z^{*}\right)^{-1}\left(1+t z^{*}\right)\right] \\
= & - \text { the } j j \text { term of }\left(1-t z^{*}\right)^{-1}\left(1-t z^{*}+z t^{*}-t z^{*} z t^{*}+1+t z^{*}\right. \\
& \left.-z t^{*}+t z^{*} z t^{*}\right)\left(1-z t^{*}\right)^{-1} \\
= & -2 \text { times the } j j \text { terms of }\left(1-t z^{*}\right)^{-1}\left(1-t t^{*}\right)\left(1-z t^{*}\right)^{-1} .
\end{aligned}
$$

Similarly for the terms $\partial G / \partial \eta^{j k}$ with $j \neq k$.

\section{REFERENCES} 1950.

1. S. Bergman, The kernel function and conformal mapping, Mathematical Surveys, No. 5,

2. - Functions of extended class in the theory of functions of several complex variables, Trans. Amer. Math. Soc. vol. 63 (1948) pp. 523-547.

3. S. Bochner, Functions of several complex variables, Princeton mimeographed notes, Princeton, 1936.

4. - Group invariance of Cauchy's formula in several variables, Ann. of Math. vol. 45 (1944) pp. 686-707.

5. R. Courant and D. Hilbert, Methoden der mathematischen Physik, vol. 2, Springer, 1937.

6. G. F. D. Duff and D. C. Spencer, Harmonic tensors on Riemannian manifolds with boundary, Ann. of Math. vol. 56 (1952) pp. 128-156.

7. L. K. Hua, On the theory of automorphic functions of a matrix variable, Amer. J. Math. vol. 66 (1944) pp. $470-488$.

8. C. C. Macduffee, The theory of matrices, Ergebnisse der Mathematik und ihrer Grenzgebiete, Chelsea, 1946.

9. J. Mitchell, The kernel function in the geometry of matrices, Duke Math. J. vol. 19 (1952) pp. 575-584.

10. Z. Nehari, Conformal mapping, McGraw-Hill, 1952.

11. F. W. Ponting and H. S. A. Potter, The volume of orthogonal and unitary space, Quarterly Journal of Mathematics vol. 19-20 (1948-1949) pp. 146-154.

12. L. Pontrjagin, Topological groups, Princeton University Press, 1946.

13. C. L. Siegel, Symplectic geometry, Amer. J. Math. vol. 65 (1943) pp. 1-86.

14. O. Veblen, Invariants of quadratic differential forms, Cambridge Tracts in Mathematics and Mathematical Physics, No. 24, 1952.

15. K. Yano and S. Bochner, Curvature and Betti numbers, Annals of Mathematics Studies, No. 32, Princeton University Press, 1953.

16. P. R. Garabedian and D. C. Spencer, A complex tensor calculus for Kähler manifolds, Acta Math. vol. 89 (1953) pp. 279-330.

17. H. E. Rauch, $A$ "Poisson" formula and the Hardy-Littlewood theorem for matrix spaces. Preliminary report, Bull. Amer. Math. Soc. Abstract 55-5-250.

18. M. Sugaward, On the theory of harmonic functions in the general Poincaré-space, J.Fac.

Sci. Imp. Univ. Tokyo. Sect. I vol. 5 (1944) pp. 1-32.

Institute for Advanced StUdy,

Princeton, N. J. 